\title{
A Green Synthesis of Grpahene Based Composite for Energy Storage Application
}

\author{
Ho Mui Yen, Jim Lim Hui Kern, Khiew Poi Sim, Chiu Wee Siong
}

\begin{abstract}
In this study, graphene-molybdenum oxide composite materials were prepared via green hydrothermal synthesis method and evaluated as supercapacitor electrodes. The morphology and structure of the composite were examined by using Scanning Electron Microscopy (SEM), Raman spectroscopy. The electrochemical performances of the composite were evaluated by cyclic voltammetry $(\mathrm{CV})$, galvanostatic chargedischarge (CD) method, and electrochemical impedance spectroscopy (EIS). The electrochemical results show that the composite electrodes possess improved specific capacitance of $122 \mathrm{~F} / \mathrm{g}$ at a scan rate of $5 \mathrm{mV} / \mathrm{s}$, which is about $22 \%$ higher that of pure graphene. Additionally, the composite electrodes exhibit good capacitive properties and a high specific energy with superior capacitive retention after 1000 cycles. In contrast to the previously reported systems that are usually complicated and costly, the present work potentially provides a readily scalable technological platform for economic mass production of energy storage devices.
\end{abstract}

Keywords: $\quad$ Supercapacitors; composite; graphene; molybdenum oxide; hydrothermal

\section{INTRODUCTION}

Global warming, air-pollution emission, rising oil prices and diminishing supply of fossil fuel have been increasingly driving the world towards the development of clean and environmentally friendly high power energy resources. Electrochemical capacitors, also known as supercapacitors, have attracted considerable attention over the past decades as the renewably-generated energy can be stored inside. Besides, it also exhibits higher power density, longer cycle life and moderate energy density. Innovative electrode material with desirable properties coupled with low cost and sustainability is the key for realizing high-performance supercapacitors.

Graphene, a two-dimensional nanosheet of graphite arranged in a honeycomb crystal structure possess excellent electrical conductivity, large theoretical surface area, good structural flexibility and superior chemical tolerance is commonly coupled with transition metal oxide that is high in specify capacity in designing the electrode material in the

Revised Manuscript Received on April 12, 2019.

Ho Mui Yen, Department of Materials Engineering, Faculty of Engineering and Technology, Tunku Abdul Rahman University College, JalanGenting Kelang, 53300 Kuala Lumpur, Malaysia.

Jim Lim Hui Kern, Department of Materials Engineering, Faculty of Engineering and Technology, Tunku Abdul Rahman University College, JalanGenting Kelang, 53300 Kuala Lumpur, Malaysia.

Khiew Poi Sim, Division of Materials, Mechanics and Structures, Center of Nanotechnology and Advanced Materials, Faculty of Engineering, University of Nottingham Malaysia Campus.43500 Semenyih, Selangor, Malaysia.

Chiu Wee Siong,Low Dimensional Materials Research Center, Department of Physics, Faculty of Science, University Malaya, 50603 Kuala Lumpur, Malaysia. supercapacitor. These include Graphene-tungsten oxide ${ }^{1}$, graphene-nickel oxide ${ }^{2}$.

A number of transition metal oxides were also reported as the electrode material in the supercapacitor includes ruthenium oxide $\left(\mathrm{RuO}_{2}\right)$, nickel oxides $(\mathrm{NiO})$, cobalt oxide $\left(\mathrm{Co}_{3} \mathrm{O}_{4}\right)$, molybdenum oxide $\left(\mathrm{MoO}_{3}\right){ }^{3}$ Molybdenum oxide is a potential candidate in the electrode material in the supercapacitor because of its layered structure that capable in ion insertion/extraction, high electrochemical performance, low electrical resistivity and excellent stability. ${ }^{4}$

The choice of the electrolyte is one of the most influential factors that could affect the overall electrochemical performance of the supercapacitor. Aqueous medium electrolytes including sulphuric acid and potassium hydroxide are of good candidates in providing higher power density due to the high ionic concentration. ${ }^{5}$ However, these aggressive electrolytes will cause severe corrosionon the energy storage devices. In this work,mild and nonflammable aqueous electrolyte (neutral sodium sulfite solution) which is beneficial to the users, the environment and the cycle life of electrodes were employed in the system.

\section{MATERIALS AND METHODS}

Herein, graphene- $\mathrm{MoO}_{3}$ composites were prepared via green hydrothermal synthesis method by using ammonium molybdate and graphene oxide as the precursors. Acetic acid was used as the reducing agent in the hydrothermal process as it is relatively non-toxic in the sense that it is not harmful to the aquatic life.

Graphene Oxide (GO) and ammonium molybdate were purchased from Ugent Tech Sdn. Bhd. and MedigeneSdn. Bhd respectively. Acetic acid (99.8\%) was purchased from Bendosen. Sodium sulfite used as the electrolyte was purchased from R\&M Chemicals.

Briefly, $5 \mathrm{mg}$ of GO and $0.6 \mathrm{~g}$ of ammonium molybdate was dissolved into $50 \mathrm{ml}$ of $5 \mathrm{M}$ acetic acid solution. The resultant mixture was then ultra-sonicated for 30 minutes to ensure a homogenous GO-dispersed solution. The homogenous dispersed solution was then transferred into the teflon-lined stainless steel autoclave and kept into the heating furnace at $180^{\circ} \mathrm{C}$ for 8 hours with ramping rate of 10 ${ }^{\circ} \mathrm{C} / \mathrm{min}$. The composite was then obtained by centrifuging the solution before proceeding to the calcination process with $650{ }^{\circ} \mathrm{C}$ for 2 hours under nitrogen atmosphere.

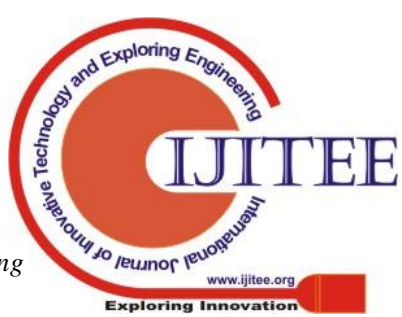


The electrode material was prepared by mixing the active material with carbon black (Ketjenblack, EC-600JD from Akzo Nobel, surface area $1400 \mathrm{~m}^{2} / \mathrm{g}$ ) and Polyvinylidenefluoride (PVDF) with the weight ratio of 80:15:5. The mixture was then diluted with N-methyl-2pyrrolidinone (NMP) before coated on $10 \mathrm{~mm}$ x $10 \mathrm{~mm}$ current collector.

The morphology and structure of the composite were examined by using Scanning Electron Microscopy (SEM), Raman spectroscopy. All the electrochemical testing was conducted with two-electrode Teflon cell in $1 \mathrm{M}$ of sodium sulfite solution $\left(\mathrm{Na}_{2} \mathrm{SO}_{3}\right)$. Cyclic voltammetry $(\mathrm{CV})$, chargedischarge testing (CD) and electrochemical impedance spectroscopy (EIS) were conducted using MetrohmAutolab PGSTAT128N potentiostat equipped with a FRA2 frequency response analyser module and FRA software.

\section{RESULTS AND DISCUSSION}

Fig. 1 shows the SEM image of the graphenemolybdenum oxide composite with the optimum combination ratio of the graphene oxide and ammonium molybdate, which is 1 to 12 . The $\mathrm{MoO}_{3}$ particles were populated and distributed uniformly on the surface of reduced graphene oxide ( $\mathrm{rGO}$ ), indicating the optimal ratio which could exhibit more superior energy storage capacity. In addition, the $\mathrm{MoO}_{3}$ sandwiched between the rGO could work as the spacer that prevents therGO from agglomeration. The mechanism of the intercalation of $\mathrm{MoO}_{3}$ between rGO could be explained in two ways. (i) The electrostatic attraction between the oxygen functional group and the molybdenum ions. ${ }^{6}$ (ii) The ammonium molybdate will forms a thin layer on the surface of the GO. ${ }^{4}$

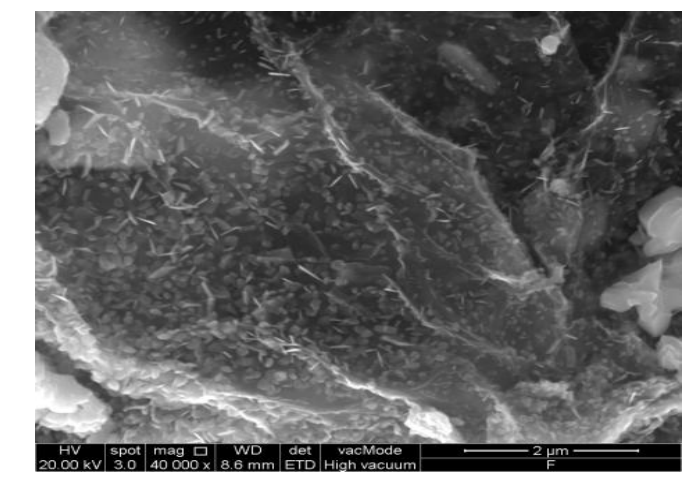

Fig. 1: SEM image of the composite. Magnification x40k

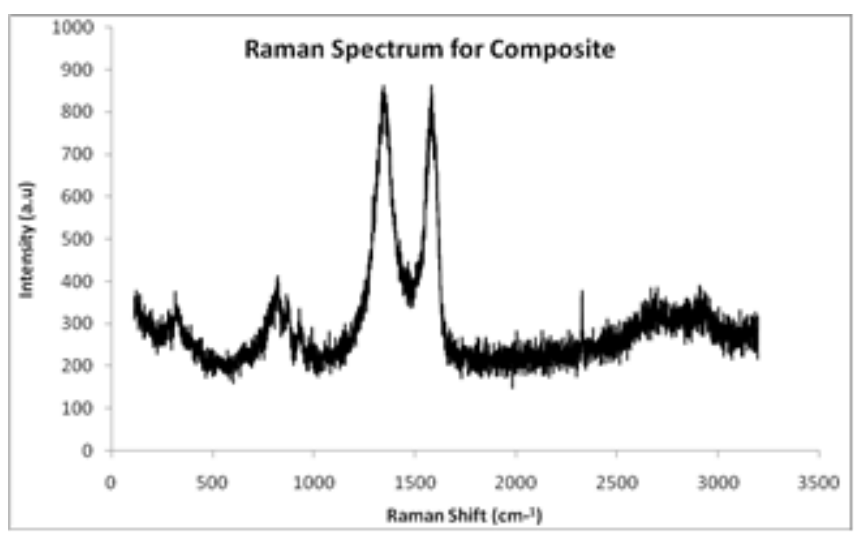

Fig. 2: Raman shift of the composite.
Fig. 2 shows the Raman spectroscopy of the composite There are 2 dominant peaks in the spectrum which are the $\mathrm{D}$ peak at $1350 \mathrm{~cm}^{-1}$ and the $\mathrm{G}$ peak at $1580 \mathrm{~cm}^{-1}$ respectively. $G$ peak is responsible to the $E_{2 g}$ phonon of the graphitic materials while the D peak is enhanced by the defects (edge defects), number of layers of the $\mathrm{rGO}^{7}$ On the other hand, there are peaks appeared at around 334,a 830, 874 and 929 $\mathrm{cm}^{-1} .334 \mathrm{~cm}^{-1}$ owning to the bending modes of the terminal $\mathrm{Mo}=\mathrm{O}$ bond. Meanwhile, 830, 874 and $929 \mathrm{~cm}^{-1}$ are attributed by either symmetric or asymmetric stretching modes of the terminal $\mathrm{Mo}=\mathrm{O}$ bond. ${ }^{8}$ The characteristic peaks of $\mathrm{MoO}_{3}$ particles and rGO appears together in a spectrum, confirming the formation of the composite without destroying the structure of rGO after the hydrothermal reduction process. ${ }^{4}$

The CV curve of the composite obtained in the potential window from $0 \mathrm{~V}$ to $1 \mathrm{~V}$ in $1 \mathrm{M}$ of $\mathrm{Na}_{2} \mathrm{SO}_{3}$ at scan rate of 5 , 20, 50 and $100 \mathrm{mV} / \mathrm{s}$ was presented in Fig. 3. The specific capacitances of the materials were calculated based on the formula in Eq. (1) where I is the applied current density, $\mathrm{dv} / \mathrm{dt}$ is the rate of change of the operating potential.

$$
C_{p}=\frac{I}{m \times \frac{d v}{d t}}
$$

The specific capacitance of the composite is $121.98 \mathrm{~F} / \mathrm{g}$ compared to the $\mathrm{rGO}$ and $\mathrm{MoO}_{3}$ which is only $95.19 \mathrm{~F} / \mathrm{g}$ and $32.90 \mathrm{~F} / \mathrm{g}$ respectively. This demonstratesthe synergistic effect of the composite between the rGO and the $\mathrm{MoO}_{3}$ which could be attributed by (i) high surface area of rGOwith excellent electrical conductivity (ii) the combination of the double layer and pseudo-capacitance that increase the total capacitance of the electrodes (iii) intercalation of the $\mathrm{MoO}_{3}$ between the $\mathrm{rGO}$, improving the effectiveness of the adsorption/desorption of ions on the rGO. $^{9}$

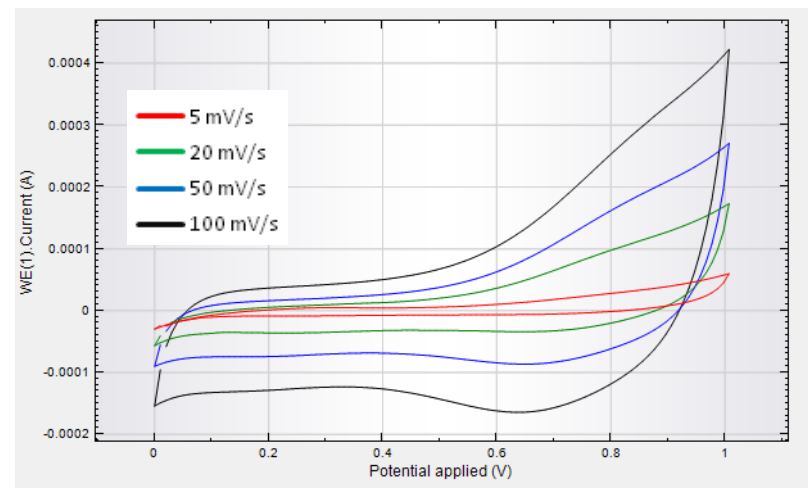

Fig. 3: Cyclic voltammograms for the composite with different scan rate in $1 \mathrm{M}$ of $\mathrm{Na}_{2} \mathrm{SO}_{3}$.

Fig. 4 shows the CV curve for the composite after 300, and 1000 cycles. After 1000 cycles, the composite exhibits $83 \%$ of reversible capacity retention. Meanwhile, the percentage of retention for $\mathrm{MoO}_{3}$ is only $62 \%$. It is expected that the $\mathrm{MoO}_{3}$ exhibits a lower percentage of retention as

the 


$$
P=\frac{E \times 3600}{\Delta t}
$$

$\mathrm{MoO}_{3}$ electrodes experience fading as the increasing chargedischarge cycles. This causes the pulverization and cracking of the material. ${ }^{4}$ which result in poorer capacity. On the other hand, the improved performance observed in this paper is attributed to the $\mathrm{rGO}-\mathrm{MoO}_{3}$ binary composite due to the fact that rGO could storemore ions on the high surface area and serve as good electrical conductors. Moreover, the rGO can work as the spacers to limit the volume expansion of the $\mathrm{MoO}_{3}$ during the charge-discharge process. ${ }^{10}$ In short, high percentage of retention indicates the good chargedischarge performance.

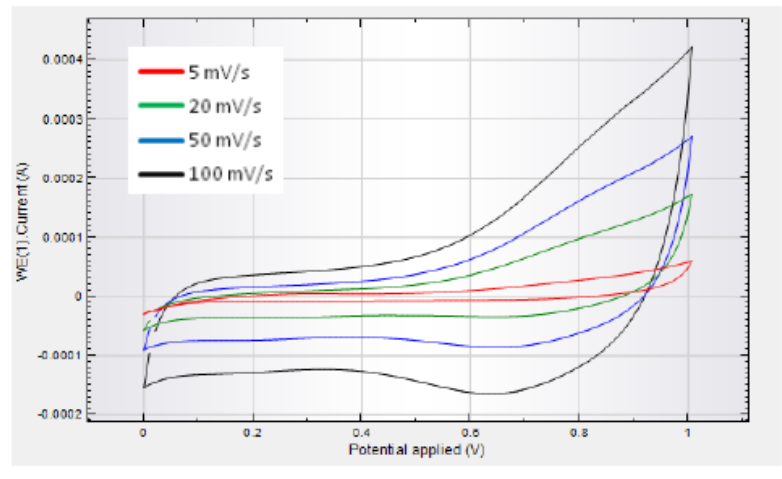

Fig. 4: Cyclic voltammograms for the composite with different number of scanning cycles at scan rate of $5 \mathrm{mV} / \mathrm{s}$ in $1 \mathrm{M}$ of $\mathrm{Na}_{2} \mathrm{SO}_{3}$.

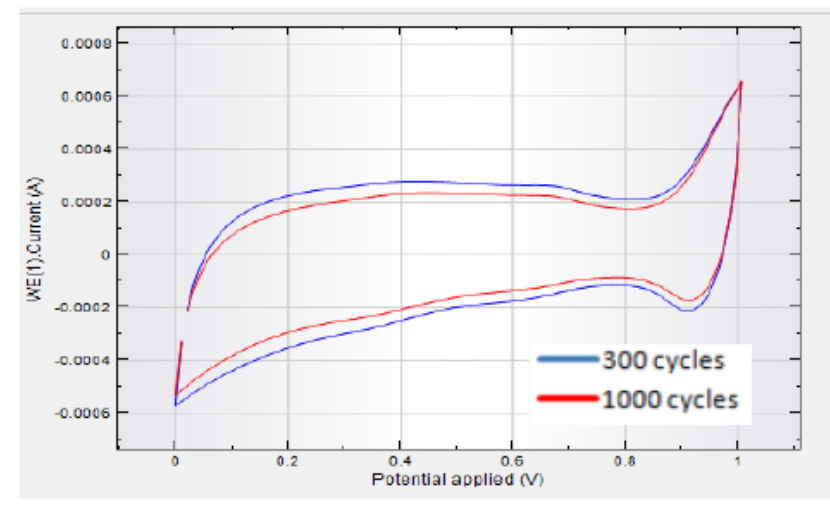

Fig. 5: Ragone chart of the materials

The energy density (E) and power density (P) of the electrode material were calculated to evaluate the electrochemical performance of the electrode material. The calculation is performed based on Eq. (2) and Eq. (3) where $\mathrm{E}$ is the energy density of the electrode in $\mathrm{Wh} / \mathrm{kg}, \mathrm{P}$ is the power density of the electrode in $\mathrm{kW} / \mathrm{kg}, \mathrm{Cs}$ is the specific capacitance of the electrode, $\Delta t$ is the total time for a sweep segment in seconds and $\nabla \mathrm{V}$ is the voltage range for a sweep segment.

$$
E=\frac{1000 \times C_{s} \nabla V^{2}}{2 \times 3600}
$$

The calculated value of energy density and power density for different scan rate were plotted in the Ragone plot as shown in Fig. 5. The composite exhibits superior energy density at $16.27 \mathrm{Wh} / \mathrm{kg}$ at power density of $306.01 \mathrm{~W} / \mathrm{kg}$. Meanwhile, the composite also exhibits excellent power density of $1323.64 \mathrm{~W} / \mathrm{kg}$ as energy density of $3.52 \mathrm{Wh} / \mathrm{kg}$. Overall, the composite demonstrate about 5 and 4 times of enhancement of the energy density and power density respectively as compared to the $\mathrm{rGO}$ and $\mathrm{MoO}_{3}$.

The IR drop of the materials was evaluated via the charge-discharge test (CD). The IR drop was calculated based on Eq. (5), where $R_{i}$ is the IR drop, $\Delta V_{2}$ is the different in the voltage and $\mathrm{I}_{\text {discharge }}$ is the current discharge.

$$
\boldsymbol{R}_{i}=\frac{\Delta \boldsymbol{V}_{2}}{I_{\text {discharge }}}
$$

The IR drop values for composite, individual $\mathrm{rGO}$ and individual $\mathrm{MoO}_{3}$ are 21.44 $\Omega, 39.67 \Omega$ and $25.10 \Omega$ respectively. It clearly shows that the composite exhibits the lowest internal resistance due to the synergetic effect of the rGO and the $\mathrm{MoO}_{3}{ }^{4}$

Electrochemical impedance spectroscopy (EIS) was conducted in order to analyze the resistance of the materials. Based on Fig. 6, it can be clearly observed that the diameter of the semicircle for $\mathrm{rGO}$ is the largest among all the materials, thus suggesting that $\mathrm{rGO}$ anode have highest contact and the charge-transfer resistance caused by the double layer capacitance is also the greatest, followed by $\mathrm{MoO}_{3}$ and finally the composite. ${ }^{11}$ As a result, the composite demonstrates the lowest charge-transfer resistance. This is attributed by the flowing factors where the present of the rGO in the composite provides good conductivity. Besides, the mesh structure of the rGOis able to hold $\mathrm{MoO}_{3}$ particlesin its micropores, preventing the $\mathrm{MoO}_{3}$ from agglomeration. As a result, it enhances the contact area between electrolyte ions and electrodes, which eventually decreases the charge-transfer resistance. ${ }^{4}$

An equivalent circuit as shown in Fig.7 was constructed in order to fit the Nyquist plot. After the simulation and fitting, it is found that the composite exhibits the lowest resistance among the electrode materials. Table 1 shows the resistance of the materials obtained from the equivalent circuit which is similar to the trend obtained in the IR drop from the CD test. 
Table 1: The resistances of different electrode materials measured in ohm $(\Omega)$.

\begin{tabular}{llll}
\hline & Composite & rGO & MoO3 \\
\hline Polarization & 2.00 & 13.50 & 3.84 \\
Solution & 0.79 & 1.81 & 0.50 \\
Total & $\mathbf{2 . 7 9}$ & $\mathbf{1 5 . 3 1}$ & $\mathbf{4 . 3 4}$ \\
\hline
\end{tabular}

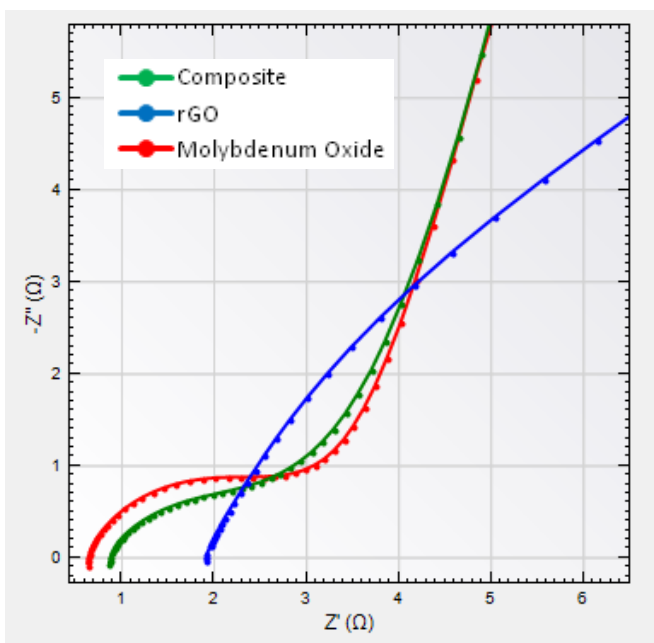

Fig. 6: Nyquist plot of the materials

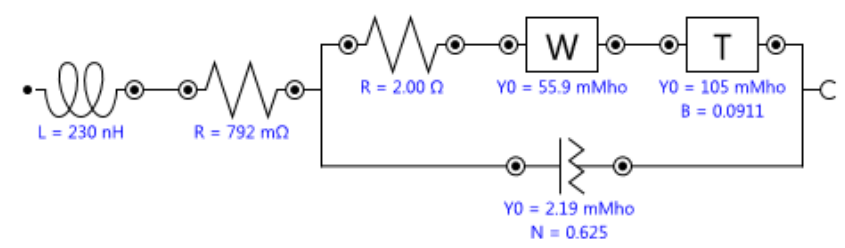

Fig. 7: Equivalent circuit for the composite to fit the Nyquist plot.

Overall, the results reveal that graphene-molybdenum oxide composite in a $\mathrm{Na}_{2} \mathrm{SO}_{3}$ neutral electrolyte exhibits excellent electrochemical performance and high cycling stability, thus displays great potential for supercapacitor applications.

\section{REFERENCES}

1. Y. Cai et al.Graphenenanosheeets-tungsten oxides composite for supercapacitor electrode (2014).

2. Y. Chen et al. Synthesis of the graphene/ nickel oxide composite and its electrochemical performance for supercapacitors (2014).

3. Y. Wang et al. Mesoporous Transition Metal Oxides for Supercapacitors. (2015).

4. Q. Tang et al. MoO2-graphene nanocomposite as anode material for lithium ion batteries (2012).

5. G. Wang et al. A review of electrode materials for electrochemical supercapacitors. (2012).

6. K. H. Seng et al. Facile Synthesis of graphene-molybdenum dioxide and its lithium storage properties. (2012)

7. V. M. Iruzun et al. Raman intensity measurements of singlewalled carbon nanotube suspensions as a quantitative technique to assess purity. (2009)
8. H. Hu et al. Surface Structures of Supported Molybdenum Oxide Catalysts. (1995).

9. E. R. Ezeigwe et al. Solvothermal synthesis of graphene$\mathrm{MnO} 2$ nanocomposites and their electrochemical behavior. (2015)

10. Y. Xu et al. High Capacity MoO2/Graphite Oxide Composite Anode for Lithium-ion Batteries. (2012).

11. Y. Li et al. Preparation of SnO2-Nanocrystal/ GrapheneNanosheets Composites and Their Lithium Storage Ability. (2010).

12. D. Zhou et al. Graphene-molybdenum oxynitride porous material with improved cyclic stability and rate capability for rechargeable lithium ion batteries. (2013). 